\title{
Using puns to study contextual influences on lexical ambiguity resolution: Evidence from eye movements
}

\author{
Heather Sheridan, Eyal M. Reingold, and Meredyth Daneman \\ University of Toronto, Mississanga, Ontario, Canada
}

\begin{abstract}
Participants' eye movements were monitored while they read sentences containing biased homographs in either a single-meaning context condition that instantiated the subordinate meaning of the homograph without ruling out the dominant meaning (e.g., "The man with a toothache had a crown made by the best dentist in town") or a dual-meaning pun context condition that supported both the subordinate and dominant meanings (e.g., "The king with a toothache had a crown made by the best dentist in town"). In both of these conditions, the homographs were followed by disambiguating material that supported the subordinate meaning and ruled out the dominant meaning. Fixation times on the homograph were longer in the single-meaning condition than in the dual-meaning condition, whereas the reverse pattern was demonstrated for fixation times on the disambiguating region; these effects were observed as early as first-fixation duration. The findings strongly support the reordered access model of lexical ambiguity resolution.
\end{abstract}

When readers encounter homographs, such as calf, pupils, and bank, how do they extract the relevant meaning from among the various meanings that are associated with a single orthographic form? Although all models of lexical ambiguity resolution agree that context allows readers to determine the correct interpretation of a homograph, there has been considerable controversy surrounding the locus of context effects (Duffy, Kambe, \& Rayner, 2001). Historically, modular or autonomous models postulated that the preceding context does not influence lexical access (e.g., Fodor, 1983; Forster, 1979). Specifically, all meanings of an ambiguous word are accessed exhaustively, regardless of context, and meaning selection is accomplished at the postlexical integration stage. In marked contrast, interactive models have argued that context influences lexical access and that such access is selective (e.g., McClelland \& Rumelhart, 1981; Morton, 1969). That is, context can constrain lexical access such that only the contextually relevant meaning is accessed.

However, following Rayner and Duffy's (1986) introduction of eye movement measurement techniques to the study of lexical ambiguity resolution, several key findings have emerged that could not be easily accounted for by either the modular or interactive models (Duffy et al., 2001). Eye movement studies manipulated various aspects of either the homographs or the context. Specifically, one critical characteristic of homographs, referred to as meaning dominance, concerns the relative frequency of the constituent meanings (Hogaboam \& Perfetti, 1975). The two types of meaning dominance profiles that were used in eye movement studies were balanced homographs, which have two approximately equally common meanings, and biased homographs, which have one highly dominant meaning and one or more subordinate meanings. In addition, the preceding context was constructed to be neutral (i.e., no disambiguating information precedes the homograph) or to instantiate either the subordinate or dominant meaning of the homograph. Typically, fixation times on homographs were compared with fixation times on unambiguous control words that were embedded in the same context. Evidence for ambiguity-related processing delays was obtained when fixation times on homographs were longer than those on control words.

A comprehensive review of the findings from eye movement studies of lexical ambiguity is beyond the scope of the present article (see Duffy et al., 2001, for such a review). Briefly, these studies demonstrated processing delays for certain combinations of preceding context and meaning dominance, but not for others. For example, when the preceding context instantiated the subordinate meaning, fixation times were longer on biased homographs than on control words (Duffy, Morris, \& Rayner, 1988). This effect was later termed the subordinate bias effect (Pacht \& Rayner, 1993; Rayner, Pacht, \& Duffy, 1994) and has been the focus of extensive empirical and theoretical efforts in the literature (Binder, 2003; Binder \& Rayner, 1999; Dopkins, Morris, \& Rayner, 1992; Duffy et al., 2001; Duffy et al., 1988; Folk \& Morris, 2003; Kambe, Rayner, \& Duffy, 2001; Pacht \& Rayner, 1993; Rayner, Binder, \& Duffy, 1999; Rayner, Cook, Juhasz, \& Frazier,

H. Sheridan, heather.sheridan@utoronto.ca 
2006; Rayner \& Frazier, 1989; Rayner et al., 1994; Sereno, 1995; Sereno, O’Donnell, \& Rayner, 2006; Sereno, Pacht, \& Rayner, 1992; Wiley \& Rayner, 2000). Placing balanced homographs in a neutral context resulted in longer fixation times on homographs than on control words (Rayner \& Duffy, 1986). Fixation times on homographs and control words did not differ for biased homographs in a preceding context that was either neutral or that instantiated the dominant meaning, and for balanced homographs in a preceding disambiguating context (Duffy et al., 1988). Both strong interactive accounts and strong modular accounts fail to adequately explain this pattern of empirical findings. Interactive models are inconsistent with the subordinate bias effect, because a preceding disambiguating context should result in selective access of the subordinate meaning without any processing delays. Modular accounts cannot explain the findings that fixation times vary depending on the type of preceding context (i.e., neutral, subordinate supported, dominant supported).

Consequently, two models were introduced to accommodate the findings obtained in eye movement studies: the reordered access model (Duffy et al., 1988) and the integration model (Rayner \& Frazier, 1989). The reordered access model asserts that lexical access is exhaustive, and the order by which meanings are accessed is determined by both preceding contextual information (i.e., meanings instantiated by the preceding context are accessed faster) and meaning dominance (i.e., more common meanings are accessed faster). When these factors or their interaction cause two or more meanings to become available at approximately the same time, competition between the meanings leads to processing delays. For example, when the preceding context supports the subordinate meaning of a biased homograph, it speeds up access to that meaning and causes the subordinate and dominant meaning to become available within the same time window, resulting in the subordinate bias effect. Unlike the reordered access model, the integration model (Rayner \& Frazier, 1989) argues that the order by which meanings are accessed is determined exclusively by meaning dominance. Rather than influencing lexical access, context is postulated to affect a postlexical integration stage by facilitating the integration of contextually relevant meanings. According to the integration model, the subordinate bias effect occurs because integration of the dominant meaning fails and the processor must wait for the subordinate meaning to become available before integration can proceed.

Although both the reordered access model and the integration model are able to accommodate most of the findings from eye movement studies and often make identical predictions, the results of several studies (Dopkins et al., 1992; Sereno, 1995; Sereno, Brewer, \& O’Donnell, 2003) and simulations (Duffy et al., 2001; Reichle, Pollatsek, \& Rayner, 2007) were more consistent with the reordered access model. In particular, a critical experiment by Dopkins et al. was designed to disentangle these two models. The key contrast in this study involved comparing fixation times across two context conditions: (1) a neutral preceding context condition in which a homograph (e.g., speaker) was preceded by context that did not support either meaning of the homograph (e.g., "Before either of us could do anything, the speaker was completely rewired by the technician") and (2) a positive context, in which preceding context supported the subordinate meaning but was consistent with the dominant meaning (e.g., "Inaudible as a result of the static, the speaker was completely rewired by the technician"). In both conditions, the homographs were followed by disambiguating material that clearly supported the subordinate meaning and ruled out the dominant meaning.

Dopkins et al. (1992) argued that the reordered access model and integration model would predict a different pattern of fixation times both on the homograph and on the disambiguating region. Specifically, the reordered access model would predict a facilitation of lexical access of the subordinate meaning in the positive context condition. Consequently, in this condition, the dominant and subordinate meanings of the homograph might be retrieved at about the same time, resulting in greater lexical competition and longer fixation times on the homograph. Given that the subordinate meaning has been accessed in the positive context but not in the neutral condition, the reordered access model would also predict faster processing of the disambiguating material in that condition than in the neutral context condition. Dopkins et al. further contended that the integration model would not predict these differences, because the preceding contexts in both conditions were consistent with the dominant meaning; therefore, in both contexts, readers should initially integrate the dominant meaning and then experience delays when they are forced to integrate the subordinate meaning upon encountering the disambiguating material.

Dopkins et al. (1992) reported that, consistent with the reordered access model but not with the integration model, fixation times in the disambiguating region were longer in the neutral context than in the positive context. However, in contrast to the prediction of the reordered access model, gaze durations on the homographs were not significantly longer in the positive than in the neutral condition, although there was a 9-msec numerical trend in that direction. Thus, although the approach introduced by Dopkins et al. allowed for a strong empirical differentiation between the two competing models, the results reported by these authors, although clearly favoring the reordered access model, failed to demonstrate the complete pattern predicted by this model. Another difficulty in interpreting the findings reported by Dopkins et al. stems from the large differences between the sentence frames in which the homographs were embedded in the positive versus the neutral context conditions. In using homographs as their own controls, Dopkins et al. avoided the complexities involved in matching homographs to unambiguous control words (this approach was also used by Rayner et al., 2006, and Rayner \& Frazier, 1989). However, this methodological advantage was achieved at the cost of producing sentence frames that might not have been equated for variables such as complexity, comprehension difficulty, and length.

Our study was designed to extend the approach introduced by Dopkins et al. (1992), in order to further distin- 
guish between the two models. We contrasted Dopkins et al.'s positive context condition, henceforth referred to as the single-meaning context (e.g., "The lawyer called the actor to talk about the suit that he filed on his behalf"), with a novel, dual-meaning (pun) context condition in which preceding context highlighted features of both the subordinate and dominant meanings without ruling out either meaning (e.g., "The lawyer called the tailor to talk about the suit that he filed on his behalf"). In both of these conditions, the homographs were followed by disambiguating material (i.e., "filed on his behalf") that clearly supported the subordinate meaning and ruled out the dominant meaning. Importantly, unlike the large differences in the context preceding the homograph in the neutral and positive conditions used by Dopkins et al., the present contrast between the dual- and single-meaning context conditions involved only a single word change between sentence frames.

Despite this similarity between the sentence frames in the present experiment, the reordered access model predicts a difference in the pattern of fixation times across conditions. Specifically, the reordered access model predicts that contextual support for the subordinate meaning in the single-meaning condition should facilitate lexical access of the subordinate meaning such that it is more likely to compete with the dominant meaning, resulting in longer fixation times on the homograph. In contrast, the dual-meaning context provides support for both the dominant and the subordinate meaning, and consequently, in this condition, the relative advantage for the dominant meaning should be greater and the likelihood of lexical competition should be reduced. In addition, as compared with the single-meaning condition, the greater relative emphasis on the dominant meaning in the dual-meaning condition would be expected to lead to a larger proportion of trials in which the subordinate interpretation was not considered until the disambiguating region, resulting in longer fixation times in this region. In marked contrast, for both the homograph and the disambiguating region, the integration model would predict no fixation-time differences between the two context conditions. This is the case because the preceding context in both conditions does not rule out the dominant meaning of the homograph, so readers would be expected to always integrate the dominant meaning upon encountering the homograph and to then experience delays upon reaching the disambiguating material.

Finally, in addition to the gaze duration measure reported by Dopkins et al. (1992), we report measures of early processing (i.e., first fixation and single fixation) in order to investigate the time course of lexical ambiguity effects. Such measures are potentially informative, because the reordered access model proposes an earlier locus of contextual effects during lexical access than does the integration model, which assumes that contextual influences are postlexical. Thus, the present study was designed to empirically distinguish between two models of lexical ambiguity resolution by examining both the time course and pattern of eye movements across two highly equated context conditions and with homographs serving as their own controls.

\section{METHOD}

\section{Participants}

All 38 undergraduate students at the University of Toronto who participated were native English speakers and were given either course credit or $\$ 10$ (Canadian) per hour. All had normal or corrected-to-normal vision.

\section{Materials and Design}

Thirty-six sentence pairs were created such that the preceding context highlighted either two meanings (dual-meaning pun condition; e.g., "The lawyer called the tailor to talk about the suit that he filed on his behalf") or a single meaning (single-meaning condition; e.g., "The lawyer called the actor to talk about the suit that he filed on his behalf") of a homograph (e.g., "suit"). The Appendix contains a complete list of the sentence pairs. In each dual-meaning sentence, one supported meaning was dominant (e.g., "suit" = clothing), the other was subordinate (e.g., "suit" = lawsuit), and the preceding context was consistent with either interpretation of the homograph. The single-meaning version always highlighted a semantic feature of the subordinate meaning of the homograph without ruling out the dominant meaning. The preceding context for the single-meaning versions of the sentences differed from that for the corresponding dual-meaning sentence by only a single word (e.g., "actor" vs. "tailor"), and the changed word and the homograph were separated by at least 4 words ( 6.5 intervening words, on average). In both versions, the homograph was followed by neutral material ( $2-6$ words; average $=2.8$ ). The neutral material was followed by a disambiguating region (2-8 words; average $=4.3$ ) that was consistent only with the subordinate meaning. The selection of the 36 noun-noun homographs that were included in the sentence pairs was based on the results of norming data collected from an independent sample of 82 participants. The norming procedure involved the participants' writing the first word that came to mind in response to a list of homographs and unambiguous filler words. The meaning supported in the 36 single-meaning sentences had a probability range of $0-.2$ and a mean of .07. The dominant meaning of the homographs had a probability range of .8-1 and a mean of .93. The homographs had a mean word length of 4.67 and a mean Francis and Kučera (1982) word frequency count of 37.8 occurrences per million.

The experimental sentences spanned one to two lines. The homograph, neutral region, and disambiguating region were always presented on the last line, and the homograph was never the first or second word on that line. All participants read a total of 81 sentences, including 9 practice trials, 36 experimental sentences, and 36 filler sentences. The filler sentences and the practice trials did not contain any puns or homographs. The order of the sentences was determined randomly for each participant. Each participant read either the dual- or single-meaning version of each sentence pair, and the presentation of sentence versions was counterbalanced across participants.

\section{Apparatus and Procedure}

Eye movements were measured with an SR Research EyeLink 1000 system. Following calibration, gaze-position error was less than $0.5^{\circ}$. The sentences were displayed on a 19 -in. ViewSonic monitor. All letters were lowercase (except when capitals were appropriate) and in a monospaced Courier font. The text was presented in black $\left(4.7 \mathrm{~cd} / \mathrm{m}^{2}\right)$ on a white background $\left(56 \mathrm{~cd} / \mathrm{m}^{2}\right)$. Viewing distance was $75 \mathrm{~cm}$ from the monitor, and 2.8 characters equaled $1^{\circ}$ of visual angle. Participants were instructed to read the sentences for comprehension. After reading each sentence, they pressed a button to end the trial and proceed to the next sentence. To ensure that participants were reading for comprehension, about $25 \%$ of the sentences were followed by multiple-choice comprehension questions. The average accuracy rate was $95.4 \%$. Following the eyetracking portion of the study, participants were given a vocabulary test to ensure that they understood both meanings of the homographs used. Participants correctly identified both meanings $94.6 \%$ of the time. 


\section{RESULTS}

The following measures were used to compare fixation times on the homographs in the single-meaning sentences versus the dual-meaning (pun) sentences: (1) first-fixation duration (i.e., the duration of the first forward fixation on the homograph, regardless of the number of subsequent fixations on the homograph), (2) single-fixation duration (i.e., the gaze duration value for the subset of trials in which there was only one first-pass fixation on the homograph), (3) gaze duration (i.e., the sum of all the consecutive first-pass fixations on the homograph, before a saccade to another word), and (4) go-past time (i.e., the sum of all fixations from the first fixation on the homograph up to and including the fixation prior to the reader moving past the homograph to a later part of the sentence). In addition to these homograph measures, we analyzed first-fixation duration and go-past time for both the neutral region and the disambiguating region. Go-past time for the disambiguating region was the sum of all fixations, starting with the first fixation in this region and ending when the reader pressed the button to remove the sentence from the screen.

Trials were excluded from the analysis if the first fixation on the homograph was longer than $750 \mathrm{msec}$ or shorter than $100 \mathrm{msec}$ ( $4 \%$ of trials were removed); the overall probabilities for single fixation, immediate refixation, and skipping of the homograph were $.65, .06$, and .25 , respectively. For each participant and item, average fixation times were calculated for both the single- and dual-meaning context conditions. For each of the mea- sures, planned comparisons by participants and by items were performed across the two context conditions. Table 1 presents the means and standard errors of the different measures and the corresponding $t$ test results.

In line with the predictions of the reordered access model, the measures of processing time on the homograph were significantly longer in the single-meaning condition than in the dual-meaning condition. As is shown in Table 1, this effect was significant for first fixation, single fixation, and gaze duration. It was marginally significant for go-past time by participants and nonsignificant for go-past time by items. Likely reflecting a spillover effect, the first fixation on the neutral region was also larger in the single-meaning condition than in the dual-meaning condition (this effect was significant by participants and marginally significant by items). However, overall go-past time in the neutral region was not significantly different across conditions. In marked contrast, in the disambiguating region the reverse pattern of results occurred. Fixation times were longer for the dual-meaning condition than for the single-meaning condition, and this was true for the very first fixation in the disambiguating region, as well as for the overall go-past time measure.

\section{DISCUSSION}

In considering the implications of the present findings for models of lexical ambiguity resolution, it is important to examine the overall pattern of results. Specifically, we observed that fixation times on the homograph were longer in the single-meaning condition than in the dual-meaning

Table 1

Average Fixation Time Measures (in Milliseconds) for the Homograph, Neutral Region, and Disambiguating Region, by Context Condition

\begin{tabular}{|c|c|c|c|c|c|c|}
\hline \multirow[b]{3}{*}{ Measure } & \multicolumn{4}{|c|}{ Fixation Time } & \multirow[b]{3}{*}{ Difference } & \multirow[b]{3}{*}{ Significance } \\
\hline & \multicolumn{2}{|c|}{$\begin{array}{l}\text { Single-Meaning } \\
\text { Sentence }\end{array}$} & \multicolumn{2}{|c|}{$\begin{array}{c}\text { Dual-Meaning } \\
\text { Sentence } \\
\end{array}$} & & \\
\hline & $M$ & $S E$ & $M$ & $S E$ & & \\
\hline \multicolumn{7}{|l|}{ Homograph } \\
\hline First fixation & 231 & 5.62 & 221 & 3.94 & 10 & $\begin{array}{l}t_{1}=2.26, p<.05 \\
t_{2}=2.46, p<.05\end{array}$ \\
\hline Single fixation & 233 & 6.06 & 223 & 4.14 & 10 & $\begin{array}{l}t_{1}=2.20, p<.05 \\
t_{2}=2.74, p<.05\end{array}$ \\
\hline Gaze duration & 254 & 8.63 & 234 & 5.66 & 20 & $\begin{array}{l}t_{1}=2.80, p<.01 \\
t_{2}=2.90, p<.01\end{array}$ \\
\hline Go-past time & 348 & 22.96 & 309 & 12.98 & 39 & $\begin{array}{l}t_{1}=1.99, p=.05 \\
t_{2}=1.34, p=.19\end{array}$ \\
\hline \multicolumn{7}{|l|}{ Neutral Region } \\
\hline First fixation & 230 & 5.87 & 217 & 6.51 & 13 & $\begin{array}{l}t_{1}=2.13, p<.05 \\
t_{2}=1.96, p=.06\end{array}$ \\
\hline Go-past time & 505 & 33.43 & 493 & 26.18 & 12 & $\begin{array}{l}t_{1}=0.41, p=.68 \\
t_{2}=0.57, p=.58\end{array}$ \\
\hline \multicolumn{7}{|c|}{ Disambiguating Region } \\
\hline First fixation & 224 & 5.37 & 239 & 4.88 & -15 & $\begin{array}{l}t_{1}=3.32, p<.01 \\
t_{2}=2.44, p<.05\end{array}$ \\
\hline Go-past time & 1,797 & 118.92 & 2,087 & 143.33 & -290 & $\begin{array}{l}t_{1}=3.10, p<.01 \\
t_{2}=2.78, p<.01\end{array}$ \\
\hline
\end{tabular}

Note-For the $t$ tests shown above, $d f$ for $t_{1}=37$ and $d f$ for $t_{2}=35$. The means and standard errors shown are based on the by-participants analyses. 
condition, whereas the reverse pattern was demonstrated for fixation times in the disambiguating region. Both of these contextual effects were evident early, as was indicated by significant differences in first-fixation duration. Building on the approach introduced by Dopkins et al. (1992), this experiment yielded a pattern of results that provides strong support for the reordered access model (Duffy et al., 1988), and the obtained findings cannot be explained by the current version of the integration model (Rayner \& Frazier, 1989).

The reordered access model accommodates well both the time course and the pattern of fixation-time differences across contexts. Specifically, in the dual-meaning, but not in the single-meaning, condition, preceding contextual support for the dominant meaning decreases the likelihood that the subordinate meaning would be accessed at the same time as the dominant meaning, resulting in reduced lexical competition and shorter fixation times on the homograph. Conversely, the higher likelihood of accessing and selecting the subordinate meaning in the single-meaning condition reduces the chance that readers will need to revise their interpretation of the sentence upon entering the disambiguating region, producing shorter fixation times in this region. In addition to explaining the pattern of fixation-time differences on the homograph and the disambiguating region, the reordered access model is also consistent with the fast-acting nature of these effects, which is indicated by the significant contextual influences on first-fixation durations. Given the temporal characteristics of saccadic programming in reading, in order for a variable to influence first-fixation duration, it must exert at least part of its influence early during a fixation (Sereno \& Rayner, 2003). Consequently, consistent with the assumptions of the reordered access model, the present first-fixation findings provide evidence that the temporal locus of contextual influences on the processing of homographs overlaps with early lexical processing (Reichle et al., 2007; Sereno et al., 2003).

In marked contrast, as explained previously, the current formulation of the integration model (Rayner \& Frazier, 1989) would predict no differences across the dual- and single-meaning conditions. This prediction follows from the fact that the preceding context in both conditions is consistent with the dominant meaning. Consequently, according to the integration model, in both conditions readers would be expected to initially integrate the dominant meaning and then to revise this interpretation upon reaching the disambiguating region. Clearly, these predictions of the integration model are incompatible with the present findings. The integration model would have to incorporate the assumption that, whereas the dominant meaning of the homograph is selected in both conditions, postlexical integration of this meaning is easier in the dual-meaning condition than in the single-meaning condition, and integration processes are sufficiently fast-acting for this effect to be manifested in first-fixation durations. A further assumption that would be required is that the discrepancy between the ongoing comprehension based on the dominant meaning of the homograph, and the disambiguating material, is easier to recover from in the single-meaning than in the dual-meaning condition. However, although this assumption of easier recovery might provide a reasonable explanation for the contextual differences in go-past time on the disambiguating region, such an interpretation is not very convincing in light of the significant difference in first-fixation durations. It is difficult to see how such differential recovery rates could be fast-acting enough to be manifested in first-fixation durations. More generally, although it is possible to suggest various modifications to the integration model in order to explain individual aspects of the present findings, we are unable to envision a modification to that model that can accommodate the pattern as a whole.

Finally, the novel dual-meaning (pun) context condition that was introduced in the present study offers unique methodological advantages for studies of lexical ambiguity resolution. This is the case, because it permitted the use of homographs as their own controls while employing essentially equivalent sentence frames. The clear empirical pattern that emerged despite this rather subtle contextual manipulation strengthens the theoretical conclusions that can be derived from the present findings. Specifically, the overall pattern of findings provides strong support for the reordered access model and is very problematic for the current version of the integration model. This by no means implies that postlexical integration does not play a role in mediating contextual effects on lexical ambiguity resolution. Rather, the present findings suggest that contextual influences on the processing of homographs are mediated at least in part by the modulation of lexical competition during lexical access.

\section{AUTHOR NOTE}

This research was funded by NSERC research grants to E.M.R. and M.D. and by a Canada Graduate Scholarship to H.S. We are grateful to Richard Gerrig, Barbara Juhasz, Keith Rayner, Sara Sereno, and Tessa Warren for their helpful comments on earlier versions of the article. Direct correspondence to H. Sheridan, E. M. Reingold, or M. Daneman, Department of Psychology, University of Toronto at Mississauga, 3359 Mississauga Road N. RM 2037B, Mississauga, ON, L5L 1C6 Canada (e-mail: heather.sheridan@utoronto.ca, reingold@psych.utoronto.ca, or daneman@psych.utoronto.ca).

\section{REFERENCES}

Binder, K. S. (2003). Sentential and discourse topic effects on lexical ambiguity processing: An eye-movement examination. Memory \& Cognition, 31, 690-702.

Binder, K. S., \& RAYNER, K. (1999). Does contextual strength modulate the subordinate bias effect? A reply to Kellas and Vu. Psychonomic Bulletin \& Review, 6, 518-522.

Dopkins, S., Morris, R. K., \& Rayner, K. (1992). Lexical ambiguity and eye fixations in reading: A test of competing models of lexical ambiguity resolution. Journal of Memory \& Language, 31, 461-477.

Duffy, S. A., Kambe, G., \& RAYner, K. (2001). The effect of prior disambiguating context on the comprehension of ambiguous words: Evidence from eye movements. In D. Gorfein (Ed.), On the consequences of meaning selection: Perspectives on resolving lexical ambiguity (pp. 27-43). Washington, DC: American Psychological Association.

Duffy, S. A., Morris, R. K., \& Rayner, K. (1988). Lexical ambiguity and fixation times in reading. Journal of Memory \& Language, 27, 429-446.

Fodor, J. A. (1983). Modularity of mind. Cambridge, MA: MIT Press. FOLK, J. R., \& MORRIS, R. K. (2003). Effects of syntactic category as- 
signment on lexical ambiguity resolution in reading: An eye movement analysis. Memory \& Cognition, 31, 87-99.

ForSTER, K. I. (1979). Levels of processing and the structure of the language processor. In W. Cooper \& E. C. T. Walker (Eds.), Sentence processing: Psycholinguistic studies presented to Merrill Garrett (pp. 27-86). Hillsdale, NJ: Erlbaum.

Francis, W. N., \& KučERA, H. (1982). Frequency analysis of English usage: Lexicon and grammar. Boston: Houghton Mifflin.

Hogaboam, T. W., \& Perfetti, C. A. (1975). Lexical ambiguity and sentence comprehension. Journal of Verbal Learning \& Verbal Behavior, 14, 265-274.

Kambe, G., Rayner, K., \& Duffy, S. A. (2001). Global context effects on processing lexically ambiguous words: Evidence from eye fixations. Memory \& Cognition, 29, 363-372.

McClelland, J. L., \& Rumelhart, D. E. (1981). An interactive activation model of context effects in letter perception: I. An account of basic findings. Psychological Review, 88, 375-407.

MoRTON, J. (1969). Interaction of information in word recognition. Psychological Review, 76, 165-178.

PACHT, J. M., \& RAYner, K. (1993). The processing of homophonic homographs during reading: Evidence from eye movement studies. Journal of Psycholinguistic Research, 22, 251-271.

RAYNer, K., Binder, K. S., \& DufFy, S. A. (1999). Contextual strength and the subordinate bias effect: Comment on Martin, Vu, Kellas, and Metcalfe. Quarterly Journal of Experimental Psychology, 52A, 841-852.

RAYNER, K., COOK, A. E., Juhasz, B. J., \& Frazier, L. (2006). Immediate disambiguation of lexically ambiguous words during reading: Evidence from eye movements. British Journal of Psychology, 97, 467-482.

RAYNER, K., \& DUFFY, S. A. (1986). Lexical complexity and fixation times in reading: Effects of word frequency, verb complexity, and lexical ambiguity. Memory \& Cognition, 14, 191-201.
RaYNER, K., \& Frazier, L. (1989). Selection mechanisms in reading lexically ambiguous words. Journal of Experimental Psychology: Learning, Memory, \& Cognition, 15, 779-790.

Rayner, K., Pacht, J. M., \& Duffy, S. A. (1994). Effects of prior encounter and global discourse bias on the processing of lexically ambiguous words: Evidence from eye fixations. Journal of Memory \& Language, 33, 527-544.

Reichle, E. D., Pollatsek, A., \& Rayner, K. (2007). Modeling the effects of lexical ambiguity on eye movements during reading. In R. P. G. van Gompel, M. H. Fischer, W. S. Murray, \& R. L. Hill (Eds.), Eye movements: A window on mind and brain (pp. 271-292). Amsterdam: Elsevier.

SERENO, S. C. (1995). Resolution of lexical ambiguity: Evidence from an eye movement priming paradigm. Journal of Experimental Psychology: Learning, Memory, \& Cognition, 21, 582-595.

Sereno, S. C., Brewer, C. C., \& O'Donnell, P. J. (2003). Context effects in word recognition: Evidence for early interactive processing. Psychological Science, 14, 328-333.

Sereno, S. C., O'Donnell, P. J., \& Rayner, K. (2006). Eye movements and lexical ambiguity resolution: Investigating the subordinatebias effect. Journal of Experimental Psychology: Human Perception \& Performance, 32, 335-350.

Sereno, S. C., Pacht, J. M., \& Rayner, K. (1992). The effect of meaning frequency on processing lexically ambiguous words: Evidence from eye fixations. Psychological Science, 3, 296-300.

SEREno, S. C., \& RAYNer, K. (2003). Measuring word recognition in reading: Eye movements and event-related potentials. Trends in $\mathrm{Cog}_{-}$ nitive Sciences, 7, 489-493.

WiLEy, J., \& RAYNER, K. (2000). Effects of titles on the processing of text and lexically ambiguous words: Evidence from eye movements. Memory \& Cognition, 28, 1011-1021.

\section{APPENDIX}

For each sentence pair, the homograph and the disambiguating region are shown in italics. The neutral region includes all the words between the homograph and the disambiguating region. The dual- and single-meaning versions of the sentence pair differed only by one word. The two alternative words are shown in the following order: dual-meaning word/single-meaning word.

1. On the night after the dance, the soccer/chess players could not stop talking about the ball because it had been so well-organized and fun.

2. She bought a new watch with the music/financial group's logo on it because the band was the perfect length for her wrist.

3. She lost her money/necklace while swimming in a river, but luckily she was near the bank of the river where the water was shallow.

4. On a Hallowe'en/Friday night at the sports stadium there were many bats for the baseball players to use.

5. After buying/giving his ducks some food, the zoo keeper noticed the bill on the injured bird.

6. When the detective approached the insect/art display in the museum, he searched carefully for bugs to avoid being overheard by his enemies.

7. To illuminate/improve her tulip conservatory, she found some new bulbs that would produce flowers of many colours.

8. All of his close friends knew that the furniture/car dealer believed that the prime minister's new cabinet should be fired for proposing tax increases.

9. The boy stopped hiking with his cows/dogs because of an injured calf that he strained earlier that day at the gym.

10. The ship's/hotel's casino was using a deck with too many cards in it.

11. Upon boarding the train, the athletes/students were told that their coach would need to be repaired before they could depart.

12. While walking across the farmland/room, his foot began to ache and he noticed the corn that looked like a large blister on his big toe.

13. The king/man with a toothache had a crown made by the best dentist in town.

14. He proposed/talked to her at the baseball game, while they were both looking toward the diamond in the field below them.

15. He wanted to open an expensive new bakery/business but he didn't have enough dough because he had spent it all at a casino.

16. The nun knew the routine/community well, so she never forgot the habit she had to wear to cover her hair.

17. We had breakfast/naps while stuck in traffic because we knew that the jam would not clear for some time 


\section{APPENDIX (Continued)}

18. The coin collector with a sweet/chipped tooth preferred this particular mint because its employees are very meticulous.

19. When preparing for her lecture/trip, the musician was inspired by the notes that she heard playing on the radio.

20. After the medieval knight found the book/bag he was looking for, he searched for a page and asked him to fetch his horse.

21. Before we could finish writing/talking about pigs, we needed to find a pen that could house the animals.

22. Upon returning to Glasgow, the Scottish plumber/teenager wanted to learn more about the pipes because this musical instrument is very popular.

23. The concerned botanist/employee rushed to the factory to check on the state of the plant because it was shutdown during the strike.

24. After careful consideration, the paranoid/hardworking farmer was still concerned that the plot was not the right kind of land on which to build his new barn.

25. My grandmother liked to play cards/music by the fireplace so that her poker could be reached for stirring the fire.

26. Late last night, the drunken sailors/guests could not find the port, because it had been consumed already.

27. After the noisy tennis/wrestling match, there were complaints about the racket made by the cheering crowd.

28. Before he could go home, the music/careful researcher had to find the records because these documents were vital for his research.

29. The lawyer asked the orthodontist/businessman to give him a retainer by providing a cheque or cash.

30. After finding a seat, the boxer's fiancée/friend looked down at the ring that was surrounded by spectators eager to see the fight.

31. After hearing about the leak in the tank, the marine/factory's expert rushed to check on the seal to see if repairs were necessary.

32. The opening of the new telescope/restaurant attracted famous celebrities because a star is always striving for publicity.

33. After moving to a new apartment, the author/painter started working on the second story of the large building, next to the elevators.

34. The lawyer called the tailor/actor to talk about the suit that he filed on his behalf.

35. To get the up-to-date numbers, the carpenter/professor had to check the table that was published in a statistics journal.

36. The storyteller tried to knit/drive while reciting her tale, but she quickly lost track of the yarn that she was telling her friends.

(Manuscript received July 15, 2008;

revision accepted for publication June 16, 2009.) 